\title{
Clinical and Radiologic Characteristics of Human Metapneumovirus Infections in Adults, South Korea
}

\author{
Hyun Jung Koo, Han Na Lee, Sang-Ho Choi, Heungsup Sung, Hwa Jung Kim, Kyung-Hyun Do
}

\begin{abstract}
Clinical features of human metapneumovirus (HMPV) infection have not been well documented for adults. We investigated clinical and radiologic features of HMPV infection in 849 adults in a tertiary hospital in South Korea. We classified patients into groups on the basis of underlying diseases: immunocompetent patients, solid tumor patients, solid organ transplantation recipients, hematopoietic stem cell transplant recipients, hematologic malignancy patients, and patients receiving long-term steroid treatment. Of 849 HMPV-infected patients, 756 had community-acquired infections, 579 had pneumonia, and 203 had infections with other pathogens. Mortality rates were highest in hematopoietic stem cell transplantation recipients $(22 \%$ at 30 days). Older age, current smoking, and underlying disease were associated with HMPV pneumonia. Body mass index and an immunocompromised state were associated with 30-day mortality rates in HMPV-infected patients. Bronchial wall thickening, ground-glass opacity, and illdefined centrilobular nodules were common computed tomography findings for HMPV pneumonia. Macronodules and consolidation were observed in $<50 \%$ of patients.
\end{abstract}

Чuman metapneumovirus (HMPV), first described I in 2001, is a common pathogen that causes acute respiratory tract infections in all age groups (1). Seropositivity for IgG against HMPV has been detected in up to $100 \%$ of persons 20 and $>65$ years of age, and reinfection is common (2-4). HMPV infection shows a seasonal pattern in the United States, Asia, and countries in Europe, and most infections occur in spring $(3,5-9)$. Although HMPV infection is usually asymptomatic or causes mild and self-limiting symptoms in young healthy adults, it can cause severe pneumonia in elderly and immunocompromised persons (10-13). HMPV infection progresses from

Author affiliations: Asan Medical Center, University of Ulsan College of Medicine, Seoul, South Korea (H.J. Koo, S-H. Choi, H. Sung, H.J. Kim, K.-H. Do); Kyung Hee University Hospital at Gangdong, Seoul, South Korea (H.N. Lee)

DOI: https://doi.org/10.3201/eid2501.181131 upper respiratory tract infection (URI) to lower respiratory tract disease in up to $60 \%$ of hematopoietic stem cell transplant (HCT) recipients (14), and mortality rates are $6 \%-40 \%(15,16)$. Moreover, $\approx 50 \%$ of patients with solid organ transplants (SOT) infected with HMPV progress to pneumonia $(13,17,18)$, and HMPV infection is frequently detected in patients with exacerbated chronic obstructive pulmonary disease (19).

Clinical characteristics such as host immunity in patients with HMPV infection and radiologic findings of HMPV pneumonia are needed for early detection of HMPV infection and for studies of HMPV pneumoniarelated outcomes $(14,20,21)$. Although a recent study of 3 long-term care facilities in Japan reported clinical and radiologic characteristics of HMPV pneumonia, that study did not assess the proportion of URIs, included only immunocompetent persons, and did not determine overall outcomes of HMPV pneumonia. Therefore, we conducted a study that included a large consecutive cohort of adults infected with HMPV and assessed the proportions of HMPV-associated URI and pneumonia in patients with various underlying disease, and laboratory findings, radiologic findings, including computed tomography (CT) images, and overall outcomes.

\section{Methods}

This retrospective consecutive cohort study covered the period January 2010-February 2016. The study was approved by the institutional review board of Asan Medical Center (approval no. 2017-0016), which waived the requirement for informed consent because of the retrospective nature of this study. During the study period, all patients who came to this hospital, regardless of whether they were in the outpatient clinic, hospitalized, or in the emergency department, and who had respiratory symptoms suggestive of URI or pneumonia underwent routine collection of nasopharyngeal swab specimens, blood cultures, or both. Testing decisions were made by the clinicians. We assessed pathogens before giving any antimicrobial drugs 
to patients with no history of treatment at another hospital. If relevant pathogens could not be identified, bronchoalveolar lavage (BAL) fluid was obtained. BAL fluid was not obtained if there was no evidence of pneumonia and symptoms were eliminated by conservative management. The decisions for laboratory testing and BAL procedures were clinician directed, and laboratory results were assessed retrospectively.

During January 2010-February 2016, a total of 15,311 patients had tests performed for respiratory virus infections. For these patients, 817 patients had multiple tests because of multiple different episodes ( $>$ a 2 -month interval between tests); 591 patients had 2 tests, 149 had 3 tests, 46 had 4 tests, 15 had 5 tests, 10 had 6 tests, 3 had 7 tests, 1 had 8 tests, and 2 had 9 tests. The total number of tests was 16,489 . If a patient was infected more than once with HMPV during the study period, we used only the first episode for analysis. We thoroughly reviewed electronic medical records of patients, and their clinical characteristics; immune status, such as transplant history and steroid or immunosuppressant use; presence of other pathogens; length of hospital stay; and clinical course, such as admission to an intensive care unit; and death.

\section{Definitions}

Community-acquired infection indicated respiratory infection detected in a person in a community without a history of hospitalization or living in a long-term care facility within the previous 14 days. Hospital-acquired infection was defined as respiratory infection that occurred $\geq 48$ hours after hospital admission with new onset respiratory symptoms. We assessed respiratory virus infection in nasopharyngeal secretions and BAL fluid by using a multiplex reverse transcription PCR and a Seeplex RV 15 ACE Detection Kit (March 2010-October 2013) or an Anyplex II RV 16 Detection Kit (November 2013-August 2017) (both from Seegene Inc., http://www.seegene.com).

Patients positive for HMPV, but with no evidence of pneumonia by chest radiographs or CT images, were defined as having URI. Patients who had new pulmonary infiltrates by chest radiographs or CT images and HMPV in nasopharyngeal samples or BAL fluid were defined as having pneumonia. Patients with other pathogens in nasopharyngeal or blood samples within 2 days of diagnosis of HMPV infection were defined as co-detection positive for another pathogen (14). Long-term steroid use was defined as steroid treatment $(\geq 5 \mathrm{mg} / \mathrm{d}$ of prednisolone or an equivalent drug) for $>6$ months because of an underlying condition or disease (Appendix Table 1, https://wwwnc. cdc.gov/EID/article/25/1/18-1131-App1.pdf), such as adrenal insufficiency, interstitial lung disease, or asthma. The mean dose of steroid was $1.80 \mathrm{mg} / \mathrm{kg} / \mathrm{d}$ (range $0.53-$ $2.02 \mathrm{mg} / \mathrm{kg} / \mathrm{d}$ ).

\section{Radiologic Evaluation}

For patients given a diagnosis of HMPV infection, we evaluated the presence of pneumonic infiltrates on chest radiographs to detect pneumonia. We also evaluated bilaterality and the number of involved zones (total of 6 zones; i.e., right and left upper, middle, and lower zones).

We performed CT examinations by using 16- or 64-detector CT scanners (SOMATOM Sensation 16; Siemens Medical Solutions, https://www.siemens.com/global/de/home.html; and LightSpeed VCT; General Electric Healthcare, https://www. gehealthcare.com). We reviewed all axial and coronal CT images on the picture archiving and communication system by using the mediastinal (width, 450 hounsfield units [HUs]; level, $50 \mathrm{HUs}$ ), lung (width, 1,500 HUs; level, $-700 \mathrm{HUs}$ ), and bone (width, 1,000 HUs; level, $200 \mathrm{HUs}$ ) window settings.

We assessed CT findings for distribution of parenchymal abnormalities (number of involved lobes and bilaterality); the presence and extent of centrilobular nodules; consolidation; ground-glass opacities; and the presence of macronodules, bronchial wall thickening, bronchiectasis, lymphadenopathy, and pleural effusion. CT patterns were defined on the basis of the glossary of terms for thoracic imaging (22). All CT results were reviewed in consensus by 2 chest radiologists ( 1 with 2 years of experience and 1 with 15 years of experience) in thoracic imaging. Results were independently reviewed by a third radiologist to evaluate the reliability of the $\mathrm{CT}$ findings (percent extent of centrilobular nodules, consolidation, and groundglass opacities).

\section{Statistical Analysis}

Patients were subgrouped according to underlying conditions: immunocompetent patients, patients with solid tumors, SOT recipients, HCT recipients, patients with hematologic malignancy (HM), and patients receiving long-term steroid treatment. We compared characteristics and outcomes of HMPV infection for each of these groups. We compared proportions of HMPV pneumonia in immunocompetent and immunocompromised patients by using the $\chi^{2}$ test. Univariate and multivariable logistic regression analyses were performed to identify clinical factors associated with HMPV pneumonia and the 30-day mortality rate in HMPV-infected patients. Body mass index (BMI) was analyzed as a continuous variable. We assessed interobserver agreement for CT findings by determining intraclass correlation coefficients with $\kappa$ statistics. We compared categorical variables by using the $\chi^{2}$ test or Fisher exact test. We compared continuous variables by using the Student $t$-test or the Mann-Whitney U test. CT findings for HMPV pneumonia without another pathogen were compared in 
immunocompetent and immunocompromised patients. A 2 -sided $p$ value $<0.05$ was defined as statistically significant. We performed all statistical analyses by using SPSS version 21.0 (SPSS Inc., https://www.ibm.com/ analytics/spss-statistics-software).

\section{Results}

\section{Patient Characteristics}

For the study period, January 2010-February 2016, we identified 850 adults infected with HMPV (Figure 1). There was 1 patient in the long-term steroid use group who had 2 episodes, and the interval between the 2 episodes was 1 year. The overall percentage of patients with HMPV infection among all those tested was 5.6\% $(850 / 15,311)$. HMPV was detected in 5.2\% $(851 / 16,489)$ of all tests. Most $(82.0 \%, 696 / 850)$ patients were given a diagnosis during March-June (Figure 2). One patient who did not undergo radiologic examination was excluded, and $579(68.2 \%)$ of the $849 \mathrm{HMPV}$-infected patients were given a diagnosis of pneumonia. For pneumonia patients, 14 patients with negative results for chest radiographs had pneumonia on the next CT scan. The percentage of pneumonia in immunocompetent patients $(72.5 \%, 333 / 459)$ was slightly higher than that for immunocompromised patients $(63.1 \%, 246 / 390)(\mathrm{p}=0.003)$.
We determined characteristics of the HMPV-infected patients (Table 1). Of the 849 patients, 459 were immunocompetent, 174 had solid tumors, 59 had a history of SOT, 9 underwent HCT, 58 had underlying HM, and 90 had a history of long-term steroid use. We provide details of their underlying diseases (Appendix Table 1). Median age of immunocompetent HMPV-infected patients was 67 years, which was higher than that for the solid tumor, SOT, HCT, and HM patient groups. Most (89\%) HMPV infections were community-acquired; the remaining infections (11\%) were nosocomial. C-reactive protein concentration $(6.5$ $\mathrm{mg} / \mathrm{dL}$ vs. $2.6 \mathrm{mg} / \mathrm{dL} ; \mathrm{p}<0.001)$, leukocyte count $\left(7.5 \times 10^{3}\right.$ cells $/ \mu \mathrm{L}$ vs. $6.5 \times 10^{3}$ cells $\left./ \mu \mathrm{L} ; \mathrm{p}=0.001\right)$, and neutrophil count $\left(5.5 \times 10^{3}\right.$ cells $/ \mu \mathrm{L}$ vs. $4.3 \times 10^{3}$ cells $\left./ \mu \mathrm{L} ; \mathrm{p}<0.001\right)$ were significantly higher in patients with pneumonia than in those without pneumonia.

Overall, 23.9\% (203/849) of patients had a pathogen other than HMPV, and 16.5\% (140/849) had pneumonia and another pathogen (Appendix Table 2). Rates for other pathogens ranged from $20 \%$ to $33 \%$ in subgroups and were highest for patients with HM $(32.8 \%, 19 / 58)$ and those receiving long-term steroid treatment $(32.2 \%, 29 / 90)$. Bacteria were the most common co-detected pathogens $(57.1 \%, 116 / 203)$.

Of the HMPV-infected patients, $65 \%$ were hospitalized for a median of 7 days (range 4 days-13 days) (Table 2). An

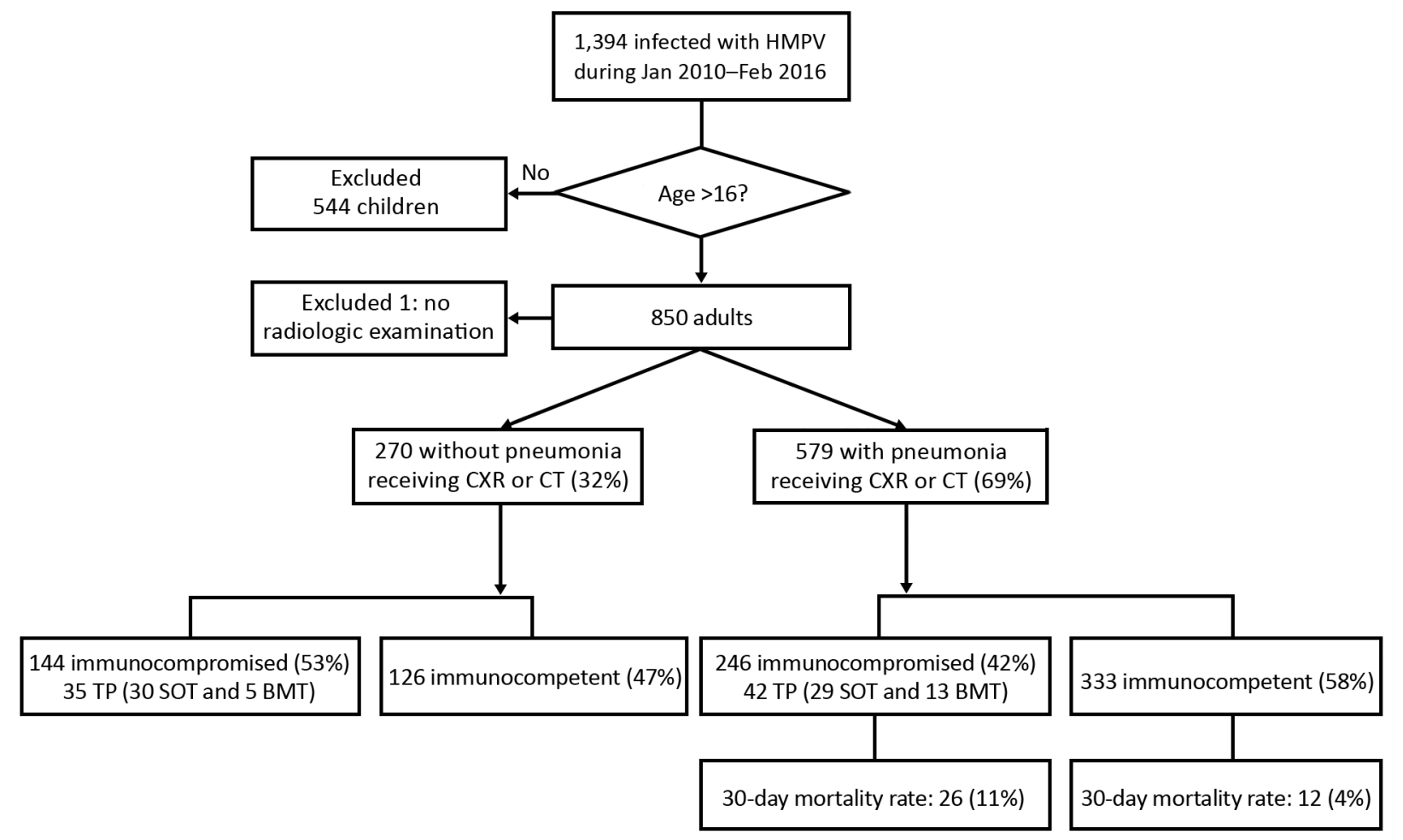

Figure 1. Flowchart for analysis of clinical and radiologic characteristics of adults with HMPV infections, South Korea. BMT, bone marrow transplant; CT, computed tomography; CXR, chest radiograph; HMPV, human metapneumovirus; SOT, solid organ transplants; TP, transplant. 

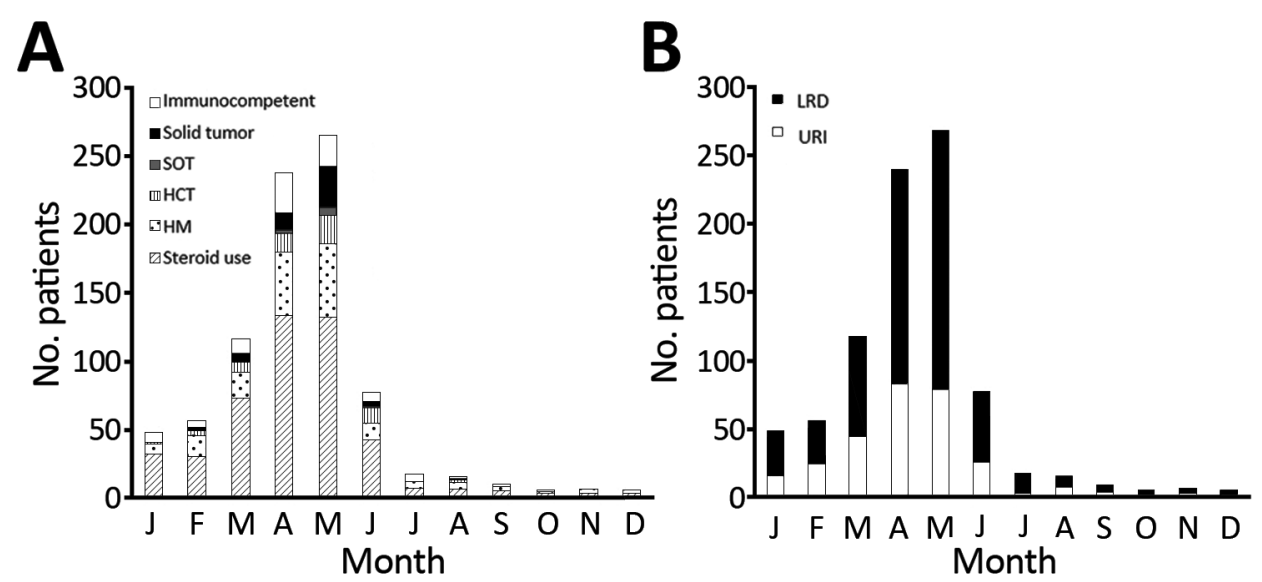

Figure 2. Monthly distribution of HMPV infection, South Korea. A) Rates of underlying diseases; B) proportions of upper respiratory tract infection and lower respiratory tract disease. HCT, hematopoietic stem cell transplantation; $\mathrm{HM}$, hematologic malignancy; HMPV, human metapneumovirus; LRD, lower respiratory tract disease; SOT, solid organ transplants; URI, upper respiratory infection. A color version of this figure is available online (http://wwwnc.cdc.gov/EID/ article/25/1/18-1131-F2.htm).

antiviral agent (oral ribavirin) was used in 129 patients, and intravenous immunoglobulin was used in 11 patients. For patients hospitalized for pneumonia, $68(8 \%)$ required admission to the intensive care unit. HCT recipients had the highest allcause 30 -day (22\%) and 90-day (33\%) mortality rates.

\section{Clinical Factors Related to HMPV Pneumonia}

We compared clinical characteristics for patients with HMPV and URI or pneumonia by using logistic regression analysis to identify factors associated with HMPV pneumonia (Table 3). Univariate analysis showed that median age was higher in patients with pneumonia than in those with URI ( 66.0 vs. 59.0 years, odds ratio [OR] 1.02, 95\% CI 1.01-1.03; $<<0.001)$. Current cigarette smoking (OR 2.10, 95\% CI 1.47-2.99; $\mathrm{p}<0.001$ ) and diabetes (OR 1.47, 95\% CI 1.08-2.01; $\mathrm{p}=0.02$ ) were also significantly associated with HMPV pneumonia. The proportion of HMPV pneumonia was higher for immunocompetent patients $(73 \%, 333 / 459)$ than for patients with solid tumors $(61 \%, 106 / 174)$ or SOT $(49 \%, 29 / 59)(p<0.001)$, However, differences for patients with HCT $(89 \%, 8 / 9)$, HM $(66 \%, 38 / 58)$, and steroid use $(70 \%, 63 / 90)$ were not statistically significant $(\mathrm{p}=0.36)$ (Figure 3$)$.

Multivariable logistic regression analysis showed that age (OR 1.02, 95\% CI 1.01-1.03; $\mathrm{p}=0.001)$ and current smoking (OR 2.07, 95\% CI 1.30-3.29; $\mathrm{p}=0.002$ ) were independently associated with HMPV pneumonia, and that diabetes showed marginal significance (OR 1.37, 95\% CI $0.96-1.95 ; p=0.08)$. The proportion of HMPV pneumonia was significantly lower for patients with solid tumors (OR $0.57,95 \%$ CI $0.38-0.84 ; \mathrm{p}=0.005$ ) or SOT (OR 0.35, $95 \%$ CI $0.19-0.65 ; \mathrm{p}=0.001)$ than for immunocompetent patients; no major differences were observed for the HCT, $\mathrm{HM}$, and steroid use groups.

\section{Clinical Factors Related to 30-Day Mortality Rates in HMPV-Infected Patients}

We found that current smoking (OR 1.92, 95\% CI $1.04-3.56$; $\mathrm{p}=0.04$ ), BMI (OR 0.89, 95\% CI 0.79-0.95; $\mathrm{p}=0.001$ ), and underlying immunocompromised status, such as patients with solid tumor, (OR 3.00, 95\% CI 1.48$6.08 ; \mathrm{p}=0.002)$, but not SOT, were significantly associated with 30-day mortality rates in HMPV-infected patients (Table 4). The association of 30-day mortality rate was highest for HCT patients (OR 7.91, 95\% CI 1.52 - 41.1; $\mathrm{p}<0.01)$. Multivariable logistic regression analysis showed that BMI (OR 0.86, 95\% CI 0.79-0.95; $\mathrm{p}=0.002)$ and underlying immunocompromised status, such as patients with solid tumor (OR 3.43, 95\% CI 1.58-7.44; $\mathrm{p}=0.002$ ), remained associated factors with 30 -day mortality rate.

\section{Radiologic Findings for HMPV Pneumonia}

We detected HMPV pneumonia radiographically for $68 \%$ $(579 / 849)$ of all patients, and $56.0 \%(324 / 579)$ of these patients had bilateral involvement. Median number of involved zones in patients with HMPV pneumonia was 3 (range 2-5). To evaluate CT findings for HMPV pneumonia, we analyzed 251 patients without another pathogen who had CT performed at the time of diagnosis (Table 5). Of these patients, 76\% (192/251) showed bilateral involvement. Of the patients with HMPV pneumonia, 69\% (174/251) had centrilobular nodules, 43\% (107/251) had consolidations, and 79\% (199/251) had ground-glass opacities (Figure 4). Macronodules $(45 \%, 114 / 251)$ and bronchial wall thickening $(88 \%, 222 / 251)$ were common findings, whereas mediastinal lymphadenopathy $(27 \%, 68 / 251)$ and pleural effusion $(22 \%, 56 / 251)$ were less common. Intraclass correlation coefficients of radiologists for CT findings (percent extents of centrilobular nodules, consolidation, and ground-glass opacities) ranged from 0.84 to $0.95(\mathrm{p}<0.001)$. The number of involved lobes was greater and pleural effusion was more frequent in immunocompromised patients than in immunocompetent patients (Figure 5; Appendix Table 3).

\section{Discussion}

We found that HMPV pneumonia in adults was associated with older age, current cigarette smoking, and underlying 
diseases. Lower BMI and immunocompromised state, bilateral lung involvement in $56 \%$ of patients, and CT scans except SOT, were associated with 30-day mortality rates showed bilateral lung involvement in $76 \%$ of patients. in HMPV-infected patients. Chest radiographs showed

Bronchial wall thickening, ground-glass opacities, and

\begin{tabular}{|c|c|c|c|c|c|c|c|}
\hline Characteristic & Immunocompetent & Solid tumors $\dagger$ & SOT & $\mathrm{HCT}$ & $\mathrm{HM}$ & Steroid use & Total \\
\hline Total & $459(54)$ & $174(20)$ & $59(7)$ & $9(1)$ & $58(7)$ & $90(11)$ & 849 \\
\hline Age, y & $67(56-75)$ & $62(53-70)$ & $56(49-62)$ & $54(32-63)$ & $60(47-67)$ & $67(52-75)$ & $64(53-73)$ \\
\hline \multicolumn{8}{|l|}{ Sex } \\
\hline M & $229(50)$ & $89(51)$ & $25(42)$ & $6(67)$ & $24(41)$ & $50(56)$ & $442(52)$ \\
\hline $\mathrm{F}$ & $230(50)$ & $85(49)$ & $34(58)$ & $3(33)$ & 34 (59) & $40(44)$ & 407 (48) \\
\hline \multicolumn{8}{|l|}{ Smoking status } \\
\hline Never & $326(71)$ & $104(60)$ & $43(73)$ & $5(56)$ & $40(69)$ & $47(52)$ & $565(67)$ \\
\hline Former & $99(22)$ & $63(36)$ & $16(27)$ & $3(33)$ & $14(24)$ & $38(42)$ & $233(6)$ \\
\hline Current & $34(7)$ & $7(4)$ & 0 & $1(11)$ & $4(7)$ & $5(6)$ & $51(27)$ \\
\hline \multicolumn{8}{|l|}{$\mathrm{BP}, \mathrm{mm} \mathrm{Hg}$} \\
\hline Systolic & $\begin{array}{c}134 \\
(120-152)\end{array}$ & $\begin{array}{c}124 \\
115-137)\end{array}$ & $\begin{array}{c}131 \\
(116-146)\end{array}$ & $\begin{array}{c}130 \\
(113-136)\end{array}$ & $\begin{array}{c}128 \\
(120-140)\end{array}$ & $\begin{array}{c}134 \\
(120-151)\end{array}$ & $\begin{array}{c}131 \\
(119-147)\end{array}$ \\
\hline Diastolic & $\begin{array}{l}(120-152) \\
80(73-90)\end{array}$ & $\begin{array}{l}(115-137) \\
80(73-88)\end{array}$ & $\begin{array}{l}(116-146) \\
82(74-92)\end{array}$ & $\begin{array}{l}(113-136) \\
88(82-90)\end{array}$ & $\begin{array}{l}(120-140) \\
82(76-90)\end{array}$ & $\begin{array}{l}(120-151) \\
82(74-90)\end{array}$ & $\begin{array}{l}(119-147) \\
80(73-90)\end{array}$ \\
\hline Temperature, ${ }^{\circ} \mathrm{C}$ & 36.6 & 36.6 & 36.4 & 36.7 & 36.5 & 36.6 & 36.6 \\
\hline & $(36.4-37.2)$ & $(36.4-37.2)$ & $(36.2-36.8)$ & $(36.5-37.7)$ & $(36.2-36.7)$ & $(36.3-36.9)$ & $(36.4-37.1)$ \\
\hline Temperature, $>37^{\circ} \mathrm{C}$ & $70(15)$ & $25(14)$ & $4(7)$ & $3(33)$ & $5(9)$ & $16(18)$ & $123(14.5)$ \\
\hline Heart rate, beats/min & $83(70-99)$ & $88(78-103)$ & $82(73-91)$ & $\begin{array}{c}100(90- \\
110)\end{array}$ & $87(77-100)$ & $93(84-106)$ & $87(74-100)$ \\
\hline $\begin{array}{l}\text { Respiratory rate, } \\
\text { breaths/min }\end{array}$ & $20(18-22)$ & $20(18-20)$ & $18(18-20)$ & $22(20-27)$ & $20(18-22)$ & $22(20-24)$ & $20(18-22)$ \\
\hline Body mass index, & 23.2 & 23.1 & 23.2 & 20.2 & 22.4 & 22.6 & 23.1 \\
\hline $\mathrm{kg} / \mathrm{m}^{2}$ & (20.4-25.9) & $(20.3-25.5)$ & $(21.3-25.1)$ & $(17.0-25.1)$ & $(20.5-25.2)$ & $(20.2-24.4)$ & $(20.4-25.5)$ \\
\hline Hypertension & $239(52)$ & $71(41)$ & $47(80)$ & $3(33)$ & $22(38)$ & $51(57)$ & $433(51)$ \\
\hline Diabetes mellitus & $157(34)$ & $42(24)$ & $44(75)$ & $4(44)$ & $20(35)$ & $29(32)$ & $296(35)$ \\
\hline \multicolumn{8}{|l|}{ Location of patients $\ddagger$} \\
\hline Outpatient clinic & 22 & 1 & 2 & 0 & 0 & 2 & 27 \\
\hline Hospitalized & 131 & 137 & 21 & 3 & 24 & 35 & 351 \\
\hline ED & 306 & 36 & 36 & 6 & 34 & 53 & 471 \\
\hline \multicolumn{8}{|l|}{ Type of infection } \\
\hline $\mathrm{CA}$ & $415(90)$ & $150(86)$ & $51(86)$ & $7(88)$ & $47(81)$ & $84(93)$ & $756(89)$ \\
\hline Nosocomial & $44(10)$ & $24(14)$ & $8(14)$ & $2(22)$ & $11(19)$ & $6(7)$ & $93(11)$ \\
\hline URI & $126(28)$ & $68(39)$ & $30(51)$ & $1(11)$ & $20(35)$ & $27(30)$ & $270(32)$ \\
\hline LRD & $333(73)$ & $106(61)$ & $29(49)$ & $8(89)$ & $38(66)$ & $63(70)$ & $579(68)$ \\
\hline Initial CRP level at & 4.3 & 6.5 & 4.6 & 10.6 & 5.8 & 4.4 & 4.9 \\
\hline $\begin{array}{l}\text { hospital admission, } \\
\mathrm{mg} / \mathrm{L}\end{array}$ & $(1.5-9.9)$ & $(2.6-13.3)$ & $(1.0-7.7)$ & $(5.5-19.7)$ & $(2.8-12.0)$ & $(1.9-4.4)$ & $(1.8-11.0)$ \\
\hline Leukocyte count at & 7.7 & 5.4 & 8.5 & 12.0 & 4.8 & 8.3 & 7.2 \\
\hline diagnosis, $\times 10^{3} / \mu \mathrm{L}$ & $(5.5-10.5)$ & $(2.8-8.7)$ & $(4.8-10.6)$ & $(6.5-13.6)$ & $(2.3-7.4)$ & $(5.7-11.5)$ & $(4.9-10.3)$ \\
\hline$>10$ & $128(28)$ & $33(19)$ & $16(27)$ & $5(56)$ & $7(12)$ & $34(38)$ & $223(26)$ \\
\hline$\leq 10$ & $331(72)$ & $141(81)$ & $43(73)$ & $4(44)$ & $51(88)$ & $56(62)$ & $626(74)$ \\
\hline Neutrophil count at & 5.5 & 3.9 & 6.1 & 7.9 & 2.9 & 6.2 & 5.1 \\
\hline diagnosis, $\times 10^{3} / \mu \mathrm{L} \S$ & $(3.4-8.3)$ & $(1.7-6.5)$ & $(3.3-8.5)$ & $(5.9-9.5)$ & $(1.3-5.1)$ & $(3.9-9.6)$ & $(3.0-8.1)$ \\
\hline$>5.0$ & $252(55)$ & $67(39)$ & $35(59)$ & $6(67)$ & $14(24)^{\prime}$ & $56(62)$ & $430(51)$ \\
\hline$\leq 5.0$ & $205(45)$ & $106(61)$ & $24(41)$ & $1(11)$ & $42(72)$ & $32(36)$ & $410(48)$ \\
\hline Lymphocyte count at & 1.3 & 0.8 & 1.0 & 2.9 & 0.8 & 1.1 & 1.1 \\
\hline diagnosis, $\times 10^{3} / \mu \mathrm{L} \S$ & $(0.9-1.8)$ & $(0.5-1.3)$ & $(0.6-1.4)$ & $(0.7-4.2)$ & $(0.4-1.7)$ & $(0.7-1.6)$ & $(0.7-1.6)$ \\
\hline$>0.7$ & $380(83)$ & $97(56)$ & $41(70)$ & $5(56)$ & $33(57)^{\prime}$ & $65(72)$ & $621(73)$ \\
\hline$\leq 0.7$ & $77(17)$ & $76(44)$ & $18(31)$ & $2(22)$ & $23(40)$ & $23(26)$ & $219(26)$ \\
\hline $\begin{array}{l}\text { Platelet count, } \times \\
10^{3} / \mu \mathrm{L}\end{array}$ & $\begin{array}{c}193 \\
(145-243)\end{array}$ & $\begin{array}{c}149 \\
(101-223)\end{array}$ & $\begin{array}{c}166 \\
(116-231)\end{array}$ & $\begin{array}{c}82 \\
(35-187)\end{array}$ & $\begin{array}{c}86 \\
(36-146)\end{array}$ & $\begin{array}{c}198 \\
(143-245)\end{array}$ & $\begin{array}{c}176 \\
(124.5-233)\end{array}$ \\
\hline Blood urea nitrogen, & 14 & 13 & 21 & 15.0 & 14.5 & 15 & 14.0 \\
\hline mg/DI & $(10-22)$ & (9-19) & $(14-31)$ & (8.5-27.0) & $(10-20.3)$ & $(11-23.5)$ & $(10.0-21.0)$ \\
\hline Creatinine, mg/dL & 0.8 & 0.8 & 1.3 & 0.9 & 0.8 & 0.8 & 0.8 \\
\hline & $(0.7-1.1)$ & $(0.6-1.0)$ & $(1.0-1.8)$ & $(0.7-1.4)$ & $(0.6-1.2)$ & $(0.6-1.1)$ & $(0.7-1.1)$ \\
\hline Procalcitonin, ng/mL & $\begin{array}{c}0.2 \\
(0.1-1.2)\end{array}$ & $\begin{array}{c}0.2 \\
(0.1-0.7)\end{array}$ & $\begin{array}{c}0.2 \\
(0.1-1.2)\end{array}$ & $\begin{array}{c}0.2 \\
(0.1-1.1)\end{array}$ & $\begin{array}{c}0.2 \\
(0.1-0.6)\end{array}$ & $\begin{array}{c}0.2 \\
(0.1-0.9)\end{array}$ & $\begin{array}{c}0.2 \\
(0.1-0.9)\end{array}$ \\
\hline
\end{tabular}

*Values are no. (\%) or median (interquartile range) unless otherwise indicated. BP, blood pressure; CA, community acquired; CRP, C-reactive protein; ED, emergency department; HCT, hematologic stem cell transplantation; HM, hematologic malignancy; HMPV, human metapneumovirus; LRD, lower respiratory tract disease; SOT, solid organ transplants; URI, upper respiratory tract infection.

†Patients with solid tumors were given chemotherapy within 6 mo.

fLocations at which patients were tested for HMPV.

$\S$ Detailed laboratory results, including neutrophil and lymphocyte counts, were unavailable for 9 patients who visited outpatient clinics 
Table 2. Co-detection of other pathogens and clinical course of patients infected with human metapneumovirus, South Korea*

\begin{tabular}{|c|c|c|c|c|c|c|c|}
\hline Characteristic & Immunocompetent & Solid tumors & SOT & $\mathrm{HCT}$ & $\mathrm{HM}$ & Steroid use & Total \\
\hline Total & $459(54)$ & $174(20)$ & $59(7)$ & $9(1)$ & $58(7)$ & $90(11)$ & 849 \\
\hline URI & $126(28)$ & $68(39)$ & $30(51)$ & $1(11)$ & $20(35)$ & $27(30)$ & $270(32)$ \\
\hline LRD & $333(73)$ & $106(61)$ & $29(49)$ & $8(89)$ & $38(66)$ & $63(70)$ & 579 (68) \\
\hline Ribavirin use & $44(10)$ & $22(13)$ & $10(17)$ & $5(56)$ & $23(40)$ & $22(24)$ & $126(15)$ \\
\hline IVIG use & $3(1)$ & 0 & $1(2)$ & 0 & $1(2)$ & $3(3)$ & $8(1)$ \\
\hline $\begin{array}{l}\text { Both ribavirin and IVIG } \\
\text { use }\end{array}$ & $2(0.4)$ & 0 & 0 & 0 & 0 & $1(1)$ & $3(0.4)$ \\
\hline \multicolumn{8}{|c|}{ Co-detection of other pathogen, $n=126$} \\
\hline URI & $17(13)$ & $18(26)$ & $10(33)$ & $1(100)$ & $10(50)$ & $7(26)$ & $63(23)$ \\
\hline LRD & $72(21)$ & $23(22)$ & $13(45)$ & $1(13)$ & $9(24)$ & $22(35)$ & $140(24)$ \\
\hline Bacteria & 48 & 20 & 16 & 1 & 11 & 20 & 116 \\
\hline Virus & 32 & 14 & 7 & 0 & 6 & 5 & 64 \\
\hline Fungi & 3 & 5 & 0 & 1 & 0 & 0 & 9 \\
\hline Bacteria and virus & 6 & 1 & 0 & 0 & 2 & 3 & 12 \\
\hline Bacteria and fungi & 0 & 1 & 0 & 0 & 0 & 0 & 1 \\
\hline Virus and fungi & 0 & 0 & 0 & 0 & 0 & 1 & 1 \\
\hline Hospital admission & $279(65)$ & $98(56)$ & $32(54)$ & $6(67)$ & $35(60)$ & $64(71)$ & $552(65)$ \\
\hline Length of hospital stay, & 6.0 & 7.0 & 6.0 & 7.0 & 10.5 & 7.0 & 7.0 \\
\hline days & $(4.0-13.0)$ & $(4.0-12.0)$ & $(4.0-14.0)$ & $(5.3-55.3)$ & $(5.8-32.5)$ & $(4.0-16.0)$ & $(4.0-13.0)$ \\
\hline ICU admission & $42(9)$ & $4(2)$ & $5(10)$ & $1(11)$ & $5(10)$ & $11(12)$ & $68(8)$ \\
\hline $\begin{array}{l}\text { All-cause mortality rate } \\
\text { at } 30 \mathrm{~d}\end{array}$ & $16(3)$ & $17(10)$ & $0(0)$ & $2(22)$ & $5(9)$ & $7(8)$ & $47(6)$ \\
\hline $\begin{array}{l}\text { All-cause mortality rate } \\
\text { at } 90 \mathrm{~d}\end{array}$ & $18(4)$ & $22(13)$ & $1(2)$ & $3(33)$ & $7(12)$ & $8(9)$ & $59(7)$ \\
\hline Overall mortality rate & $42(9)$ & $34(20)$ & $3(5)$ & $4(44)$ & $21(36)$ & $22(24)$ & $126(15)$ \\
\hline
\end{tabular}

ill-defined centrilobular nodules were common CT findings in patients with HMPV pneumonia.

Recent studies have demonstrated that low BMI was a major risk factor for hospitalization of patients with pneumonia $(23,24)$. A U-shaped relationship between BMI and pneumonia has been shown in a recent meta-analysis, in which an underweight condition $(\mathrm{BMI}<18.5)$ and morbid obesity (BMI $\geq 40$ ) are both associated with the risk for community-acquired pneumonia (25). Conversely, being overweight and obese (BMI 25.0-39.9) were major factors in reducing the risk for death from pneumonia (26). In our study, the risk for HMPV pneumonia associated with BMI was not evident because only 14 patients were underweight, and there was no patient with a BMI $\geq 32$. However, lower BMI increased the mortality rate for HMPV pneumonia. This finding coincides with the obesity survival paradox, which shows a decreased pneumonia mortality rate for overweight and obese patients (26).

Although most young healthy adults with HMPV infection are asymptomatic or have mild symptoms, HMPV pneumonia can cause severe symptoms in elderly (7) and immunocompromised $(14,17,27,28)$ patients. Mortality

\begin{tabular}{|c|c|c|c|c|c|c|}
\hline \multirow[b]{2}{*}{ Characteristic } & \multirow[b]{2}{*}{ URI, $\mathrm{n}=270$} & \multirow[b]{2}{*}{ LRD, $\mathrm{n}=579$} & \multicolumn{2}{|c|}{ Univariate analysis } & \multicolumn{2}{|c|}{ Multivariable analysis } \\
\hline & & & OR $(95 \% \mathrm{Cl})$ & $p$ value & OR $(95 \% \mathrm{Cl})$ & $p$ value \\
\hline Age & $59.0(49.3-69.8)$ & $66.0(56.0-74.0)$ & $1.02(1.01-1.03)$ & $<0.001$ & $1.02(1.01-1.03)$ & 0.001 \\
\hline Male sex & $121(45)$ & $321(55)$ & $1.57(1.17-2.09)$ & 0.002 & $0.95(0.65-1.40)$ & 0.81 \\
\hline Smoking & & & & $<0.001$ & & 0.01 \\
\hline Never & $209(77)$ & $356(61)$ & 1 & & 1 & \\
\hline Former & $12(4)$ & $39(7)$ & $1.91(0.98-3.73)$ & 0.59 & $1.66(0.80-3.43)$ & 0.17 \\
\hline Current & $51(19)$ & $182(31)$ & $2.10(1.47-2.99)$ & $<0.001$ & $2.071 .30-3.29)$ & 0.002 \\
\hline Body mass index, $\mathrm{kg} / \mathrm{m}^{2}$ & $23.4(21.0-25.7)$ & $22.7(20.1-25.4)$ & $0.97(0.94-1.00)$ & 0.09 & $0.97(0.93-1.00)$ & 0.07 \\
\hline Diabetes mellitus & $79(29)$ & $217(37)$ & $1.47(1.08-2.01)$ & 0.02 & $1.37(0.96-1.95)$ & 0.08 \\
\hline Community-acquired pneumonia & $238(88)$ & $516(89)$ & $0.83(0.53-1.30)$ & 0.41 & $1.10(0.68-1.79)$ & 0.69 \\
\hline Other pathogen & $63(23)$ & $140(24)$ & $0.92(0.66-1.30)$ & 0.64 & $1.05(0.73-1.52)$ & 0.80 \\
\hline Underlying condition & & & & 0.002 & & 0.003 \\
\hline Immunocompetent & $126(47)$ & $333(58)$ & 1 & & 1 & \\
\hline Solid tumor & $68(25)$ & $106(18)$ & $0.59(0.41-0.85)$ & 0.005 & $0.57(0.38-0.84)$ & 0.005 \\
\hline SOT & $30(11)$ & $29(5)$ & $0.37(0.21-0.63)$ & $<0.001$ & $0.35(0.19-0.65)$ & 0.001 \\
\hline HCT & $1(0.4)$ & $8(1)$ & $3.03(0.38-24.45)$ & 0.30 & $3.75(0.44-31.85)$ & 0.23 \\
\hline $\mathrm{HM}$ & $20(7)$ & $38(7)$ & $0.72(0.40-1.28)$ & 0.26 & $0.79(0.43-1.45)$ & 0.45 \\
\hline Steroid use & $27(10)$ & $63(11)$ & $0.88(0.54-1.45)$ & 0.62 & $0.72(0.43-1.22)$ & 0.22 \\
\hline
\end{tabular}

*Values are no. (\%) or median (interquartile range). HT, hematologic stem cell transplantation; HM, hematologic malignancy; LRD, lower respiratory tract disease; OR, odds ratio; SOT, solid organ transplants; URI, upper respiratory tract infection. 
Table 4. Clinical characteristics associated with 30-day mortality rate for patients with human metapneumovirus, South Korea*

\begin{tabular}{|c|c|c|c|c|c|c|}
\hline \multirow[b]{2}{*}{ Characteristic } & \multirow{2}{*}{$\begin{array}{c}\text { Recovered, } \mathrm{n}= \\
802\end{array}$} & \multirow{2}{*}{$\begin{array}{l}\text { 30-day mortality } \\
\text { rate, } n=47\end{array}$} & \multicolumn{2}{|c|}{ Univariate analysis } & \multicolumn{2}{|c|}{ Multivariable analysis } \\
\hline & & & OR $(95 \% \mathrm{Cl})$ & $p$ value & OR $(95 \% \mathrm{Cl})$ & $p$ value \\
\hline Age & $63.5(53.0-73.0)$ & $67.0(55.0-77.0)$ & $(0.99-1.04)$ & 0.15 & $1.02(1.00-1.05)$ & 0.11 \\
\hline Male sex & $416(52)$ & $26(55)$ & $0.87(0.48-1.57)$ & 0.65 & & \\
\hline Smoking & & & & 0.04 & & 0.54 \\
\hline Never & 540 (67) & $25(53)$ & 1 & & 1 & \\
\hline Former & $48(6)$ & $3(6)$ & $1.35(0.39-4.63)$ & 0.63 & $0.67(0.15-3.04)$ & 0.61 \\
\hline Current & $214(27)$ & $19(40)$ & $1.92(1.04-3.56)$ & 0.04 & $1.35(0.69-2.64)$ & 0.39 \\
\hline Body mass index, $\mathrm{kg} / \mathrm{m}^{2}$ & $23.1(20.5-25.6)$ & $20.5(18.2-24.1)$ & $0.89(0.79-0.95)$ & 0.001 & $0.86(0.79-0.95)$ & 0.002 \\
\hline Diabetes mellitus & $277(35)$ & $19(40)$ & $1.29(0.71-2.35)$ & 0.41 & $1.48(0.77-0.95)$ & 0.24 \\
\hline Community-acquired pneumonia & $715(89)$ & $40(85)$ & $0.70(0.30-1.60)$ & 0.39 & $0.83(0.32-2.11)$ & 0.69 \\
\hline Other pathogen & $193(24)$ & $10(21)$ & $0.86(0.42-1.76)$ & 0.68 & $0.75(0.35-1.60)$ & 0.45 \\
\hline Underlying condition & & & & 0.02 & & 0.02 \\
\hline Immunocompetent & $443(55)$ & $16(34)$ & 1 & & & \\
\hline Solid tumor & $157(20)$ & $17(36)$ & $3.00(1.48-6.08)$ & 0.002 & $3.43(1.58-7.44)$ & 0.002 \\
\hline SOT & $59(7)$ & 0 & $0.00(0.00-0.00)$ & 1.00 & $0.00(0.00-0.00)$ & 1.00 \\
\hline $\mathrm{HCT}$ & $7(0.9)$ & $2(4)$ & $7.91(1.52-41.1)$ & 0.01 & $9.19(1.47-57.37)$ & 0.02 \\
\hline $\mathrm{HM}$ & $53(7)$ & $5(11)$ & $2.61(0.92-7.42)$ & 0.07 & $3.44(1.14-10.34)$ & 0.03 \\
\hline Steroid use & $83(10)$ & $7(15)$ & $2.34(0.93-5.85)$ & 0.07 & $2.40(0.91-6.34)$ & 0.08 \\
\hline
\end{tabular}

rates for HMPV pneumonia have been reported to be higher (up to $40 \%$ ) in HCT recipients (15). A recent meta-analysis showed that the overall HMPV mortality rate for HCT recipients was $6 \%$ and that the HMPV-associated mortality rate was 5.9 times higher in patients with pneumonia $(27 \%)$ than in patients with URI (16). In our study, although the number of HCT patients was small $(n=9)$, the mortality rate was highest for HCT recipients, and the all-cause 30day mortality rate was $22 \%$. Conversely, the proportion of SOT patients with pneumonia was lower than that for immunocompetent patients, and mortality rates were lower for SOT patients than for all other groups.

The proportion of patients with HMPV pneumonia among those with HMPV infection was smaller for the solid tumor and SOT groups than for immunocompetent patients, whereas the mortality rate was highest for HCT recipients, followed by those with HM. This finding might be explained by the fact that immunocompetent patients visited the tertiary hospital only when respiratory symptoms were not eliminated by management in a primary clinic, whereas patients about to receive organ transplants or HCT visited the tertiary hospital if they had less severe symptoms, and had more periodical follow-up visits. Therefore, the proportion of pneumonia in the immunocompetent group was high in this study.

In our study, use of ribavirin was more common in ICU admitted patients, and mortality rates were also higher in patients who received ribavirin. However, this study was not designed to analyze the efficacy of ribavirin, and ribavirin might been have used more often for treatment in severely ill patients. Thus, the effect of ribavirin use was not determined in this study.

We found that, radiologically, most patients with HMPV pneumonia had bilateral involvement; the main findings on CT images included ill-defined centrilobular

\begin{tabular}{|c|c|c|c|c|c|c|c|}
\hline \multirow[b]{2}{*}{ Characteristic } & \multicolumn{4}{|c|}{ Solid } & \multicolumn{3}{|c|}{ Steroid } \\
\hline & Immunocompetent & tumors & SOT & HCT & $\mathrm{HM}$ & use & Total \\
\hline $\begin{array}{l}\text { Patients with HMPV pneumonia } \\
\text { without another pathogen }\end{array}$ & 259 & 81 & 36 & 7 & 29 & 41 & 453 \\
\hline Patients who underwent CT scans & 138 & 43 & 26 & 3 & 23 & 18 & 251 \\
\hline Bilateral & $100(72.5)$ & $34(79.1)$ & $21(80.8)$ & $3(100)$ & $20(87.0)$ & $14(77.8)$ & $192(76)$ \\
\hline No. involved lobes & $3(2-6)$ & $5(3-6)$ & $4(2-5)$ & $6(6-6)$ & $5(3-6)$ & $4(3-5)$ & $4(2-6)$ \\
\hline Macronodule & $67(49)$ & $19(44)$ & $11(31)$ & $1(33)$ & $12(52)$ & $4(22)$ & $114(45)$ \\
\hline Patients with centrilobular nodules & $101(73)$ & $28(65)$ & $17(47)$ & $2(67)$ & $13(57)$ & $13(72)$ & $174(69)$ \\
\hline Extent of centrilobular nodules & $10(5-30)$ & $10(0-20)$ & $10(0-20)$ & $20(10-20)$ & $10(0-20)$ & $10(0-23)$ & $10(5-20)$ \\
\hline Patients with consolidation & $57(41)$ & $20(47)$ & $13(50)$ & 0 & $8(35)$ & $9(50)$ & $107(43)$ \\
\hline Extent of consolidation & $0(0-10)$ & $0(0-15)$ & $3(0-10)$ & $0(0-0)$ & $0(0-10)$ & $8(0-20)$ & $0(0-10)$ \\
\hline Patients with ground-glass opacity & $109(79)$ & $38(88)$ & $21(81)$ & $2(67)$ & $15(65)$ & $14(78)$ & $199(79)$ \\
\hline Extent of ground-glass opacity & $10(10-20)$ & $10(10-30)$ & $10(10-23)$ & $10(10-10)$ & $10(5-20)$ & $18(9-33)$ & $10(10-20)$ \\
\hline Bronchial wall thickening & $121(88)$ & $35(81)$ & $25(96)$ & $3(100)$ & $1(4)$ & $17(94)$ & $222(88)$ \\
\hline Bronchiectasis & $17(12)$ & $4(9)$ & $4(15)$ & $1(33)$ & $3(13)$ & $5(28)$ & $34(14)$ \\
\hline Cavitation & $1(0.7)$ & 0 & 0 & 0 & 0 & $1(6)$ & $2(1)$ \\
\hline Lymphadenopathy & 40 (29) & $14(33)$ & $6(23)$ & $0(0)$ & $2(9)$ & $6(33)$ & $68(27)$ \\
\hline Pleural effusion & $24(17)$ & $15(35)$ & $7(27)$ & $1(33)$ & $5(22)$ & $4(22)$ & $56(22)$ \\
\hline
\end{tabular}

*Values are no. (\%) or median (interquartile range) unless otherwise indicated. CT, computed tomography; HCT, hematologic stem cell transplantation;

$\mathrm{HM}$, hematologic malignancy; LRD, lower respiratory tract disease; SOT, solid organ transplants; URI, upper respiratory tract infection. 


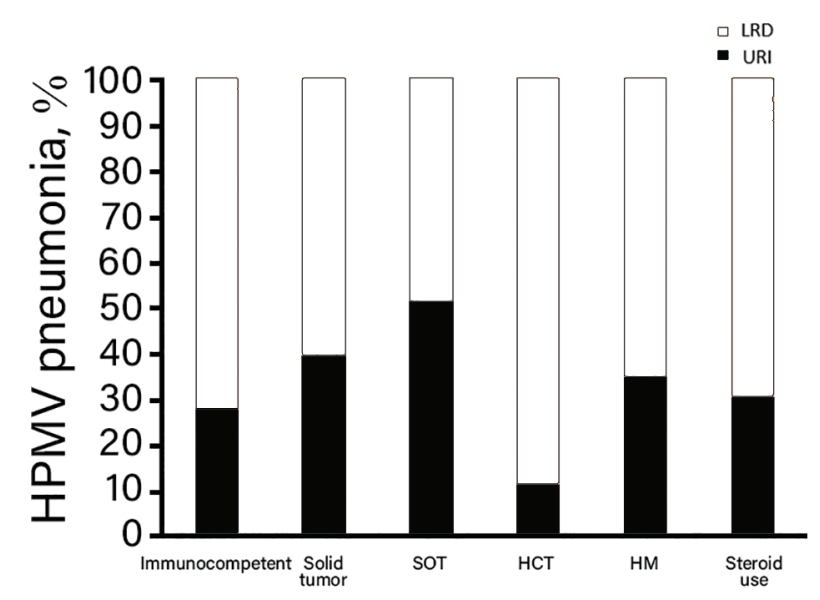

Figure 3. Proportion of HMPV-infected patients with various underlying diseases having HMPV pneumonia, South Korea. $\mathrm{HCT}$, hematopoietic stem cell transplantation; HM, hematologic malignancy; HMPV, human metapneumovirus; LRD, lower respiratory tract disease; SOT, solid organ transplants; URI, upper respiratory tract infection.

nodules, ground-glass opacities, and irregular nodular consolidations. These findings suggested bronchitis and bronchiolitis and were consistent with those of previous studies (28-32). Findings of bronchial wall thickening, bronchiolitis, and centrilobular nodules were likely caused by pathogenesis of HMPV pneumonia, which affects the airways and lung epithelia and induces inflammatory cascades (3335). Because these CT findings are also observed for bacterial pneumonia, they might be insufficient for excluding the possibility of another pathogen. CT scans of patients infected with HMPV but without another pathogen showed that the number of involved lobes and the frequency of pleural effusion were greater in the immunocompromised patients than in the immunocompetent patients.

This study had several limitations. First, patients with mild symptoms and no definite lesions by chest radiography might not undergo CT examinations. Therefore, patients with mild pneumonia that is not clearly visible on chest radiographs and who have not undergone CT scans might be classified as being in the URI group. Second, patients with low virus loads might be undetected because the Seeplex PCR has a sensitivity of $88 \%$ and the Anyplex test has a sensitivity of $96 \%$ for detecting HMPV infection (36). In addition, we did not routinely assess virus loads in specimens by using quantitative methods. Therefore, the effect of virus load on development of HMPV pneumonia could not be assessed, although virus load was shown not to be a major factor for HCT recipients (14). Third, we routinely checked patients who had respiratory infection symptoms by using nasopharyngeal swab specimens or blood cultures in our hospital. However, the decision for testing was made by clinicians, and patients with subtle respiratory symptoms might not be identified.

In conclusion, we report clinical and radiologic findings for HMPV infection in patients with various immune states. About half of these patients, even those who were immunocompetent, had HMPV pneumonia. Older age and current smoking were strongly associated with HMPV pneumonia, and the mortality rate was high in HCT recipients. CT showed that bilateral bronchial wall thickening, ground-glass opacities, and ill-defined centrilobular nodules were common.

H.J.K., H.N.L., S.H.C., and K.-H.D. designed the study; H.J.K., H.N.L., S.H.C, H.S., and K.-H.D. wrote the article; H.J.K., H.N.L., S.H.C, H.S., and K.-H.D. conducted the study; H.J.K., H.J. Kim, and H.S. developed new reagents or analytic tools; and H.J.K. and H.J. Kim analyzed data.

\section{About the Author}

Dr. Koo is a diagnostic radiologist in the Chest and Cardiovascular section, Department of Radiology and Research Institute of Radiology, Asan Medical Center, University of Ulsan College of Medicine, Seoul, South Korea. Her research interests include emerging respiratory infections.
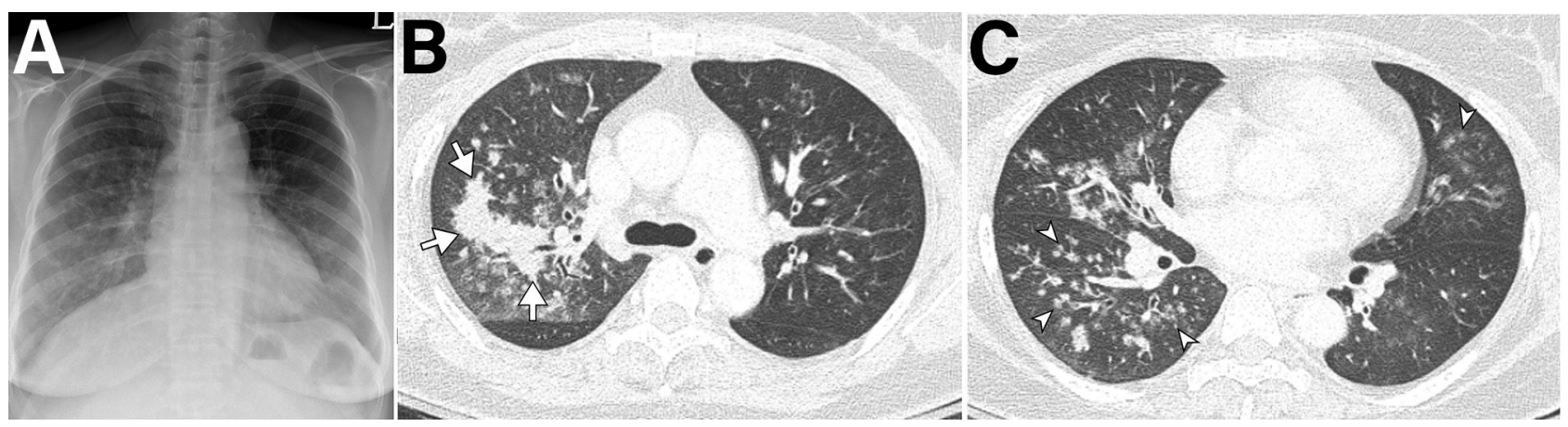

Figure 4. Imaging of 55-year-old immunocompetent woman with human metapneumovirus pneumonia, South Korea. A) Initial chest radiograph showing ill-defined patchy and nodular ground-glass opacities in the right lung and left lower lung zone. B, C) Chest computed tomography showing irregular nodular consolidation (arrows in panel B) and multiple ill-defined centrilobular nodular opacities (arrowheads in panel C) with mild bronchial wall thickening. Five days later, the lesions had resolved completely. 

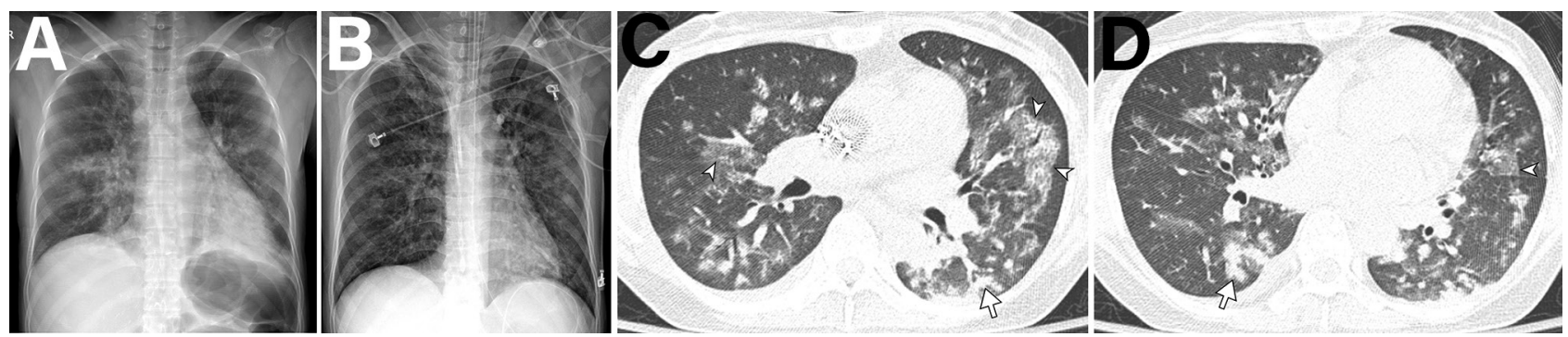

Figure 5. Imaging of 38-year-old woman in South Korea who underwent liver transplantation 2 months earlier, followed by immunosuppressant therapy, who visited the emergency department because of dyspnea, chills, and fever. A) Initial chest radiograph showing bilateral peribronchial infiltration in the central areas of both lungs and minimal pleural effusion. B) Five days later, the patient was admitted to the intensive care unit and underwent intubation because of progressive dyspnea. Radiograph showed extensive bilateral peribronchial nodular infiltrates. C, D) Computed tomography performed on the same day as in panel B showing multifocal peribronchial nodular consolidation (arrows), ground-glass opacities (arrowheads), and irregular small nodules, along with small amounts of bilateral pleural effusion. The patient was given ribavirin and prophylactic antimicrobial drugs and recovered after 10 days in the intensive care unit.

\section{References}

1. van den Hoogen BG, de Jong JC, Groen J, Kuiken T, de Groot R, Fouchier RA, et al. A newly discovered human pneumovirus isolated from young children with respiratory tract disease. Nat Med. 2001;7:719-24. http://dx.doi.org/10.1038/89098

2. Lu G, Gonzalez R, Guo L, Wu C, Wu J, Vernet G, et al. Large-scale seroprevalence analysis of human metapneumovirus and human respiratory syncytial virus infections in Beijing, China. Virol J. 2011;8:62. http://dx.doi.org/10.1186/1743-422X-8-62

3. Kahn JS. Epidemiology of human metapneumovirus. Clin Microbiol Rev. 2006;19:546-57. http://dx.doi.org/10.1128/CMR.00014-06

4. Lüsebrink J, Wiese C, Thiel A, Tillmann RL, Ditt V, Müller A, et al. High seroprevalence of neutralizing capacity against human metapneumovirus in all age groups studied in Bonn, Germany. Clin Vaccine Immunol. 2010;17:481-4. http://dx.doi.org/10.1128/ CVI.00398-09

5. Boivin G, De Serres G, Hamelin ME, Côté S, Argouin M, Tremblay G, et al. An outbreak of severe respiratory tract infection due to human metapneumovirus in a long-term care facility. Clin Infect Dis. 2007;44:1152-8. http://dx.doi.org/ 10.1086/513204

6. El Chaer F, Shah DP, Kmeid J, Ariza-Heredia EJ, Hosing CM, Mulanovich VE, et al. Burden of human metapneumovirus infections in patients with cancer: risk factors and outcomes. Cancer. 2017;123:2329-37. http://dx.doi.org/10.1002/cncr.30599

7. Falsey AR, Erdman D, Anderson LJ, Walsh EE. Human metapneumovirus infections in young and elderly adults. J Infect Dis. 2003;187:785-90. http://dx.doi.org/10.1086/367901

8. Kim S, Sung H, Im HJ, Hong SJ, Kim MN. Molecular epidemiological investigation of a nosocomial outbreak of human metapneumovirus infection in a pediatric hemato-oncology patient population. J Clin Microbiol. 2009;47:1221-4. http://dx.doi.org/ 10.1128/JCM.01959-08

9. Te Wierik MJ, Nguyen DT, Beersma MF, Thijsen SF, Heemstra KA. An outbreak of severe respiratory tract infection caused by human metapneumovirus in a residential care facility for elderly in Utrecht, the Netherlands, January to March 2010. Euro Surveill. 2012;17:17.

10. Englund JA, Boeckh M, Kuypers J, Nichols WG, Hackman RC, Morrow RA, et al. Brief communication: fatal human metapneumovirus infection in stem-cell transplant recipients. Ann Intern Med. 2006;144:344-9. http://dx.doi.org/10.7326/ 0003-4819-144-5-200603070-00010

11. Sumino KC, Agapov E, Pierce RA, Trulock EP, Pfeifer JD, Ritter JH, et al. Detection of severe human metapneumovirus infection by real-time polymerase chain reaction and histopathological assessment. J Infect Dis. 2005;192:1052-60. http://dx.doi.org/10.1086/432728

12. Walsh EE, Peterson DR, Falsey AR. Human metapneumovirus infections in adults: another piece of the puzzle. Arch Intern Med. 2008;168:2489-96. http://dx.doi.org/10.1001/ archinte.168.22.2489

13. Koo HJ, Lee HN, Choi SH, Sung H, Oh SY, Shin SY, et al. Human Metapneumovirus infection: pneumonia risk factors in patients with solid organ transplantation and computed tomography findings. Transplantation. 2018;102:699-706. http://dx.doi.org/10.1097/ TP.0000000000001965

14. Seo S, Gooley TA, Kuypers JM, Stednick Z, Jerome KR, Englund JA, et al. Human metapneumovirus infections following hematopoietic cell transplantation: factors associated with disease progression. Clin Infect Dis. 2016;63:178-85. http://dx.doi.org/10.1093/cid/ciw284

15. Renaud C, Xie H, Seo S, Kuypers J, Cent A, Corey L, et al. Mortality rates of human metapneumovirus and respiratory syncytial virus lower respiratory tract infections in hematopoietic cell transplantation recipients. Biol Blood Marrow Transplant. 2013;19:1220-6. http://dx.doi.org/10.1016/j.bbmt.2013.05.005

16. Shah DP, Shah PK, Azzi JM, El Chaer F, Chemaly RF. Human metapneumovirus infections in hematopoietic cell transplant recipients and hematologic malignancy patients: a systematic review. Cancer Lett. 2016;379:100-6. http://dx.doi.org/10.1016/ j.canlet.2016.05.035

17. Niggli F, Huber LC, Benden C, Schuurmans MM. Human metapneumovirus in lung transplant recipients: characteristics and outcomes. Infect Dis (Lond). 2016;48:852-6. http://dx.doi.org/ 10.1080/23744235.2016.1204661

18. Noel N, Rammaert B, Zuber J, Sayre N, Mamzer-Bruneel MF, Leruez-Ville M, et al. Lower respiratory tract infection in a renal transplant recipient: do not forget metapneumovirus. Case Rep Transplant. 2012;2012:353871.

19. Martinello RA, Esper F, Weibel C, Ferguson D, Landry ML, Kahn JS. Human metapneumovirus and exacerbations of chronic obstructive pulmonary disease. J Infect. 2006;53:248-54. http://dx.doi.org/10.1016/j.jinf.2005.11.010

20. Renaud C, Campbell AP. Changing epidemiology of respiratory viral infections in hematopoietic cell transplant recipients and solid organ transplant recipients. Curr Opin Infect Dis. 2011; 24:333-43. http://dx.doi.org/10.1097/QCO.0b013e3283480440

21. Weigt SS, Gregson AL, Deng JC, Lynch JP III, Belperio JA. Respiratory viral infections in hematopoietic stem cell and 
solid organ transplant recipients. Semin Respir Crit Care Med. 2011;32:471-93. http://dx.doi.org/10.1055/s-0031-1283286

22. Hansell DM, Bankier AA, MacMahon H, McLoud TC, Müller NL, Remy J. Fleischner Society: glossary of terms for thoracic imaging. Radiology. 2008;246:697-722. http://dx.doi.org/10.1148/ radiol.2462070712

23. Blumentals WA, Nevitt A, Peng MM, Toovey S. Body mass index and the incidence of influenza-associated pneumonia in a UK primary care cohort. Influenza Other Respi Viruses. 2012;6:28-36. http://dx.doi.org/10.1111/j.1750-2659.2011.00262.x

24. Phung DT, Wang Z. Risk of pneumonia in relation to body mass index in Australian aboriginal people. Epidemiol Infect. 2013; 141:2497-502. http://dx.doi.org/10.1017/S0950268813000605

25. Phung DT, Wang Z, Rutherford S, Huang C, Chu C. Body mass index and risk of pneumonia: a systematic review and metaanalysis. Obes Rev. 2013;14:839-57. http://dx.doi.org/10.1111/ obr. 12055

26. Nie W, Zhang Y, Jee SH, Jung KJ, Li B, Xiu Q. Obesity survival paradox in pneumonia: a meta-analysis. BMC Med. 2014;12:61. http://dx.doi.org/10.1186/1741-7015-12-61

27. Fisher CE, Preiksaitis CM, Lease ED, Edelman J, Kirby KA, Leisenring WM, et al. Symptomatic respiratory virus infection and chronic lung allograft dysfunction. Clin Infect Dis. 2016;62:313-9. http://dx.doi.org/10.1093/cid/civ871

28. Franquet T, Rodríguez S, Martino R, Salinas T, Giménez A, Hidalgo A. Human metapneumovirus infection in hematopoietic stem cell transplant recipients: high-resolution computed tomography findings. J Comput Assist Tomogr. 2005;29:223-7. http://dx.doi.org/10.1097/01.rct.0000157087.14838.4c

29. Kamboj M, Gerbin M, Huang CK, Brennan C, Stiles J, Balashov S, et al. Clinical characterization of human metapneumovirus infection among patients with cancer. J Infect. 2008;57:464-71. http://dx.doi.org/10.1016/j.jinf.2008.10.003

30. Karimata Y, Kinjo T, Parrott G, Uehara A, Nabeya D, Haranaga S, et al. Clinical features of human metapneumovirus pneumonia in non-immunocompromised patients: an investigation of three long-term care facility outbreaks. J Infect Dis. 2018;218:868-75. http://dx.doi.org/10.1093/infdis/jiy261

31. Shahda S, Carlos WG, Kiel PJ, Khan BA, Hage CA. The human metapneumovirus: a case series and review of the literature. Transpl Infect Dis. 2011;13:324-8. http://dx.doi.org/10.1111/ j.1399-3062.2010.00575.x

32. Syha R, Beck R, Hetzel J, Ketelsen D, Grosse U, Springer F, et al. Human metapneumovirus (HMPV) associated pulmonary infections in immunocompromised adults: initial CT findings, disease course and comparison to respiratory-syncytial-virus (RSV) induced pulmonary infections. Eur J Radiol. 2012;81:4173-8. http://dx.doi.org/10.1016/j.ejrad.2012.06.024

33. Kuiken T, van den Hoogen BG, van Riel DA, Laman JD, van Amerongen G, Sprong L, et al. Experimental human metapneumovirus infection of cynomolgus macaques (Macaca fascicularis) results in virus replication in ciliated epithelial cells and pneumocytes with associated lesions throughout the respiratory tract. Am J Pathol. 2004;164:1893-900. http://dx.doi.org/10.1016/S0002-9440(10)63750-9

34. Koo HJ, Lim S, Choe J, Choi SH, Sung H, Do KH. Radiographic and CT features of viral pneumonia. Radiographics. 2018;38: 719-39. http://dx.doi.org/10.1148/rg.2018170048

35. Chang A, Masante C, Buchholz UJ, Dutch RE. Human metapneumovirus (HMPV) binding and infection are mediated by interactions between the HMPV fusion protein and heparan sulfate. J Virol. 2012;86:3230-43. http://dx.doi.org/10.1128/ JVI.06706-11

36. Kim HK, Oh SH, Yun KA, Sung H, Kim MN. Comparison of Anyplex II RV16 with the xTAG respiratory viral panel and Seeplex RV15 for detection of respiratory viruses. J Clin Microbiol. 2013;51:1137-41. http://dx.doi.org/10.1128/JCM.02958-12

Address for correspondence: Kyung-Hyun Do, Department of Radiology and Research Institute of Radiology, University of Ulsan College of Medicine, Asan Medical Center, 88, Olympic-ro 43-gil, Songpa-gu, Seoul 05505, South Korea; email: dokh@amc.seoul.kr

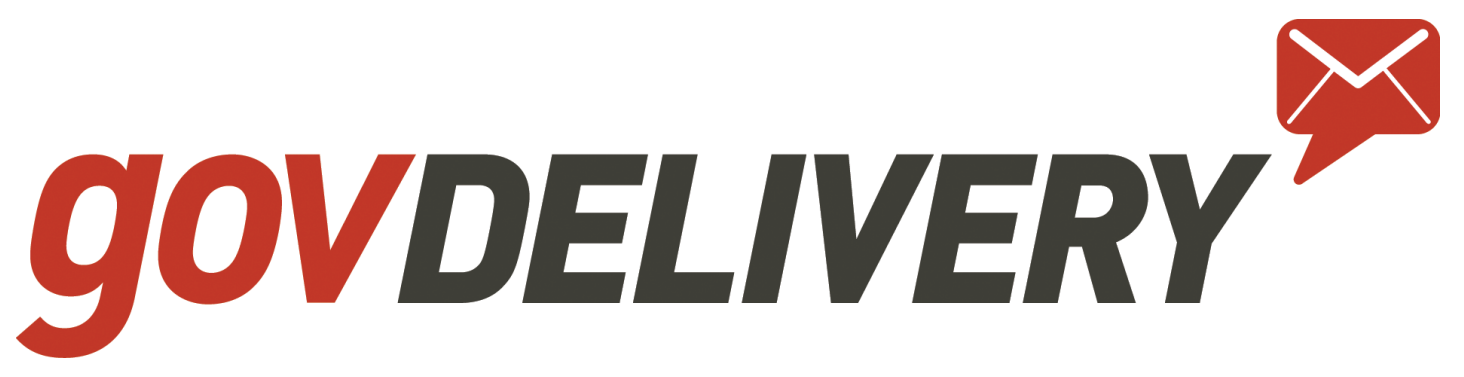

Manage your email alerts so you only receive content of interest to you. Sign up for an online subscription: wwwnc.cdc.gov/eid/subscribe.htm 